\title{
Patient reported outcome in posttraumatic pituitary deficiency: results from The Danish National Study on posttraumatic hypopituitarism
}

\author{
Marianne Klose, Kirstine Stochholm', Jurgita Janukonyté1, \\ Louise Lehman Christensen ${ }^{2}$, Arieh S Cohen ${ }^{4}$, Aase Wagner ${ }^{5}$, Peter Laurberg ${ }^{3}$, \\ Jens Sandahl Christiansen ${ }^{1}$, Marianne Andersen ${ }^{2}$ and Ulla Feldt-Rasmussen \\ Department of Medical Endocrinology, PE2131, Copenhagen University Hospital, Rigshospitalet, Blegdamsvej 9, \\ DK-2100 Copenhagen, Denmark, 'Department of Internal Medicine and Endocrinology, Aarhus University Hospital, \\ Aarhus, Denmark, ${ }^{2}$ Department of Medical Endocrinology, Odense University Hospital, Odense, Denmark, \\ ${ }^{3}$ Clinical Mass Spectrometry Unit, Section for Newborn Screening and Hormone Analysis, Department of Clinical \\ Biochemistry, Statens Serum Institut and ${ }^{4}$ Neuroradiologic Unit, Department of Diagnostic Radiology, \\ Copenhagen University Hospital, Rigshospitalet, Copenhagen, Denmark and ${ }^{5}$ Department of Medical \\ Endocrinology, Aalborg University Hospital, Aalborg, Denmark
}

\author{
Correspondence \\ should be addressed \\ to $\mathrm{M}$ Klose \\ Email \\ klose@rh.dk
}

\begin{abstract}
Objective: Posttraumatic pituitary hormone deficiency is often suggested. The impact of these predominantly mild and often irreproducible deficiencies on outcome is less clear. The aim of the present study was to describe patient reported outcome in a national a priori unselected cohort of patients with traumatic brain injury (TBI) in relation to deficiencies identified upon pituitary assessment.

Design and methods: We conducted a nationwide population-based cohort study. Participants were Danish patients with a head trauma diagnosis recorded in the Danish Board of Health diagnostic code registry; 439 patients (and 124 healthy controls) underwent assessment of anterior pituitary function 2.5 years (median) after TBI. Questionnaires on health-related quality of life (QoL) (SF36, EuroQoL-5D, QoL assessment of GH deficiency in adults) and fatigue (MFI-20) were completed in parallel to pituitary assessment.

Results: Patients with TBI had significant detriments in QoL. Impairment (mainly physical scales) related to pituitary deficiency, although only partially confirmed after adjustment for demographic differences. Hypogonadotropic hypogonadism related to several QoL scores. Increasing impairments were observed with declining total testosterone concentrations (men), but not free testosterone concentrations or any other hormone concentrations. Total testosterone was not independently related to impaired QoL and fatigue, after adjustment for demographics, and treatment with antidiabetics, opioids, antidepressants, and anticonvulsants.

Conclusions: Only a very limited relationship between pituitary hormone deficiencies and QoL/fatigue was demonstrated. Due to the dominating influence of concurrent comorbidities, pituitary deficiencies were not independently related to QoL/fatigue. Causality is still to be shown, and whether substitution therapy could be of additional relevance in selected patients needs to be proven.




\section{Introduction}

Traumatic brain injury (TBI) is one of the most common causes of death and disabilities worldwide. The majority of cases are mild (90\%), and while most recover within 3 months, many are left with significant functional limitations or psychosocial morbidity. Thus, impairments such as depression, anxiety, fatigue, sexual dysfunction, pain, sleep disorders, cognitive dysfunction, and decreased health-related quality of life (QoL) are all commonly described in TBI survivors.

Within the past 15 years, pituitary hormone deficiency was often described in the immediate and long-term followup of TBI patients, which led to recommendations that patients with head trauma should be considered for pituitary assessment (1). However, recent data from large cohorts raised doubt about the evidence behind such recommendations, as they were unable to confirm the previously reported high prevalence of pituitary dysfunction $(2,3,4)$. Although severe hypopituitarism may certainly present as a complication to brain injury, mostly mild and often irreproducible deficiencies have been reported.

The impact of post-TBI pituitary insufficiencies has been less addressed. Hypopituitary symptoms highly overlap those observed in TBI patients, and there are data to suggest that hypopituitarism may worsen TBI morbidity including QoL $(5,6,7,8)$, and functional outcome (9), whereas conflicting results exist as to cognitive function impairment $(9,10)$.

Questioning the impact of the predominantly mild and often irreproducible pituitary hormone deficiencies found by mostly single pituitary function assessment, the objective of this study was to examine patient reported outcome in a national a priori unselected cohort of wellcharacterized patients with TBI, in relation to deficiencies upon single pituitary assessment.

\section{Subject and methods}

\section{Participants}

We recruited patients hospitalized in 2008 with a head trauma diagnosis recorded in the Danish Board of Health diagnostic code registry, aged 18-65 years, with total length of hospitalization $\geq 24 \mathrm{~h} ; 2014$ patients were identified from a background population of 5.5 million DK citizens. By retrospective chart review, 856 patients were eligible for inclusion, meeting the following criteria: loss of consciousness, amnesia, or cranial/cerebral imaging abnormalities, and exclusion criteria published previously (4). Of these patients, 463/856 (54\%) patients accepted and underwent pituitary assessment according to the protocol median 2.5 (range 1.1-4.0) years after the trauma. CT scans available from the time of admission were classified by a radiologist (A W). Baseline traumarelated data registered were based on the clinical description at hospitalization and at the time of endocrine testing. Data on current medication were obtained at the time of endocrine testing.

For comparison, 124 healthy gender, BMI, and age group-matched controls recruited by newspaper advertisement or web-based recruiting (www.forsogsperson.dk) were included. Cutoff values for male hypogonadism and for the $250 \mu \mathrm{g}$ synacthen test were based on healthy cohorts as previously reported $(11,12)$.

The protocol was approved by the local ethics committee (J. nr H-B-2008-122). All participants or their closest relatives gave written informed consent before enrolment.

\section{Pituitary assessment and diagnostic criteria}

Pituitary hormone assessment was performed at one of four participating centers. All patients were scheduled for baseline evaluation and growth hormone $(\mathrm{GH})$ and adrenocorticotropin (ACTH) stimulation tests by either an insulin tolerance test (ITT) or a pyridostigmin-GHreleasing hormone (PD-GHRH) test + a $250 \mu \mathrm{g}$ synacthen test. A GHRH-arginine test was performed in all cases of contraindications to the ITT or PD-GHRH test. The study was made as part of a larger protocol, which included dual testing in some patients. Pituitary status in the present study relates to biochemical results from the first test performed. Assessment of all anterior pituitary axes was performed in 439/463 (95\%) patients, whereas 21 (5\%) refrained from GH testing, and three patients were tested by baseline evaluation only; 13/439 patients had missing biochemical data (one free thyroxine $\left(\mathrm{T}_{4}\right) ; 12$ prolactin). The final cohort thus included 426 patients.

Local cutoff values were calculated from healthy controls. Cutoff values for the $250 \mu \mathrm{g}$ synacthen test, ITT, PD-GHRH and GHRH-arg tests, and prolactin were defined by the lower $90 \%$ confidence limits (CL) of the 2.5 th percentile (mean -1.96 s.D.) for each hormone.

Similar definitions were used for $\mathrm{fT}_{4}$ and testosterone to define central hypothyroidism and male hypogonadism, given thyrotropin (TSH) and luteinizing hormone (LH) below their upper 90\% CLs of their 97.5th percentile respectively. Central hypogonadism in premenopausal 
women was defined by amenorrhea or oligomenorrhea and low estradiol, while in postmenopausal women by LH/follicle-stimulating hormone (FSH) below the lower reference limits given by the local laboratory as very few healthy postmenopausal women were included for appropriate definition.

Thus, secondary adrenal deficiency was defined as peak cortisol $<398 \mathrm{nmol} / \mathrm{l}$ (ITT) or as $30 \mathrm{~min}$ cortisol $<510 \mathrm{nmol} / 1$ (synacthen test), hypoprolactinemia as prolactin $<61 \mathrm{mU} / \mathrm{l}$, and secondary hypothyroidism as $\mathrm{fT}_{4}$ $<11.1 \mathrm{pmol} / \mathrm{l}$ (TSH $<4.5 \mathrm{mU} / \mathrm{l})$. GH deficiency was defined as peak GH $<1.9 \mu \mathrm{g} / \mathrm{l}$ in response to ITT, or as peak GH $<10.3,2.9$, and $1.2 \mu \mathrm{g} / \mathrm{l}$ in response to PD-GHRH/argGHRH in normal weight, overweight, and obese subjects respectively. Male hypogonadotropic hypogonadism was defined as a serum total testosterone $<12 \mathrm{mmol} / \mathrm{l}$ (waist circumference $<102 \mathrm{~cm}$ ) and $<7.5 \mathrm{mmol} / 1$ (waist circumference $\geq 102 \mathrm{~cm}$ ) given $\mathrm{LH}<6 \mathrm{U} / \mathrm{l}$.

\section{Assays}

GH was analyzed by chemiluminescence immunoassay (DPC Immulite 2000; Siemens, calibrated against WHO International Standard 98/574). Lower and upper limits of quantification were 0.05 and $40 \mu \mathrm{g} / 1$ respectively, with analytic sensitivity of $0.01 \mu \mathrm{g} / \mathrm{l}$. The local intra-assay coefficient of variations (CV) were 8 and $9 \%$ at concentrations of 2.6 and $6 \mu \mathrm{g} / \mathrm{l}$ respectively. Plasma cortisol, TSH, $\mathrm{fT}_{4}, \mathrm{LH}, \mathrm{FSH}$, and estradiol were analyzed by electrochemiluminescence immunoassay (Modular-E module; Roche, $\mathrm{GmbH})$. The local long-term total assay variations were cortisol 8 and $9 \%$ at concentrations of 116 and $978 \mathrm{nmol} / 1$ respectively; TSH 4 and $6 \%$ at concentrations of 0.9 and $5 \mathrm{mU} / \mathrm{l}$ respectively; $\mathrm{fT}_{4} 7 \%$ at concentrations of 12 and $30 \mathrm{pmol} / \mathrm{l} ; \mathrm{LH} 7 \%$ at concentrations of 5 and $70 \mathrm{U} / \mathrm{l} ; \mathrm{FSH} 7 \%$ at concentrations of 8 and $40 \mathrm{U} / \mathrm{l}$; estradiol 10 and $7 \%$ at a concentration of 0.2 and $2.5 \mathrm{nmol} / 1$ respectively.

Plasma prolactin was analyzed by immunofluorescence (Kryptor; Thermo Fisher, Berlin, Germany) calibrated against 3rd IS WHO 84/50; local total assay CVmax was $8 \%$ at a concentration of $120 \mathrm{mU} / \mathrm{l}$.

Analyses of total testosterone, SHBG, and free testosterone were performed at Statens Serum Institute, Copenhagen, Denmark. Proteins were precipitated using acetonitrile containing isotopically labelled internal standard. Testosterone was determined by reversed-phase chromatography with tandem mass spectrometry. Calibrators and controls were purchased from Perkin Elmer (Waltham, MA, USA). The sensitivity and the intra- and inter-assay CV were total testosterone $0.1 \mathrm{nmol} / 1,9 \%$, and
$10 \%$ respectively. SHBG levels were determined by immunofluorometric assays using an Abbot Architect. The sensitivity and the intra- and inter-assay CV were $0.1 \mathrm{nmol} / \mathrm{l}, 4 \%$, and $6 \%$ respectively. Free testosterone was calculated from the measurement of total testosterone and SHBG (13).

\section{Patient reported outcome}

Outcome questionnaires were completed in parallel to pituitary hormone assessment in patients and healthy controls. Two generic QoL questionnaires (EuroQoL-5D (EQ5D) (14) and Medical Outcomes Study 36-Item ShortForm Health Survey (SF36) (15)), and one specific for GH deficiency (QoL assessment of GH deficiency in adults (QoLAGHDA) (16)) were included. Due to the common complaint of fatigue in TBI patients, the Multidimensional Fatigue Inventory (MFI-20) (17) was added.

Furthermore, non-standardized questions were included as to whether or not patients had experienced changes in symptoms related to pituitary disease including changes of libido, erectile dysfunction, hair growth, menstrual dysfunction, tiredness, cold intolerance, sleep (amount and pattern), thirst, and nycturia. Questions were answered dichotomously.

\section{Statistical analysis}

Categorical data are presented as number (\%), continuous data as mean (s.D.) if normally distributed and otherwise as median (range). Comparison of categorical data was performed by the $\chi^{2}$ test or Fisher's exact test in tables with expected frequencies $<5$. Between-group comparisons were analyzed by unpaired $t$-tests for normally distributed data, otherwise by the Mann-Whitney $U$ test. Univariate and multivariate regression analyses were conducted to analyze the association between outcome measures (questionnaire scores) and covariates (pituitary deficiencies, hormone concentrations, demographics, trauma characteristics, and medications). Multivariate analyses were conducted with backward elimination. No interactions were identified. Statistical analyses were performed by SAS version 9.1 (SAS Institute, Inc., Cary, NC, USA). In all cases, a difference was considered significant when $P<0.05$.

\section{Results}

\section{Patient characteristics}

The population included 426 patients with full anterior pituitary function assessment (Table 1). Most patients 
Table 1 Characteristics in pituitary sufficient and deficient TBI patients respectively. Data are expressed as number (\%) unless specified otherwise.

\begin{tabular}{l}
\hline \\
\hline Gender (male) \\
Age (years) \\
BMI (kg/m ${ }^{2}$ ) \\
Trauma cause \\
Road accident \\
Fall \\
Assault \\
Other \\
Glasgow coma scale \\
Mild (GCS 13-15) \\
Moderate (GCS 9-12) \\
Severe (GCS $<9$ ) \\
Posttraumatic amnesia \\
Yes \\
Length (h) \\
Hospitalization (days) \\
Intracerebral lesions (CT) \\
Axonal injury \\
Oedema \\
Midline shift $>4$ mm \\
SAH \\
EDH \\
SDH \\
Contusions \\
Vault fractures \\
Base of skull fractures \\
Glasgow outcome score $<5$ \\
Medication (at the time of pituitary \\
testing) \\
AEDs \\
Opioids \\
Antidepressants \\
Antidiabetics \\
\end{tabular}

\begin{tabular}{c}
\hline$n$ \\
\hline 426 \\
426 \\
426 \\
426
\end{tabular}

\begin{tabular}{c}
\hline Deficient patients $(n=84)$ \\
\hline $62(74 \%)$ \\
$48.2(13.3)$ \\
$26.2(4.8)$ \\
$36(43 \%)$
\end{tabular}

\begin{tabular}{c} 
Sufficient patients $(n=342)$ \\
\hline $220(64 \%)$ \\
$43.6(14.7)$
\end{tabular}

\begin{tabular}{l}
\multicolumn{1}{c}{$\boldsymbol{P}$} \\
0.1 \\
0.01 \\
0.92
\end{tabular}

$36(43 \%)$

$32(38 \%)$

$5(6 \%)$

$11(13 \%)$

$43.6(14.7)$

0.92

(2)

412

$60(72 \%)$

$7(8 \%)$

$154(45 \%)$

0.90

$23(36)$

$24(7 \%)$

$41(12 \%)$

$7(8 \%)$
$16(19 \%)$

$234(71 \%)$

$33(10 \%)$

$62(19 \%)$

$70(84 \%)$

$0.5(0-504)$

$418 \quad 4(1-108)$

$284(85 \%)$

$0.9(0-144)$

$4(1-44)$

0.8

357

$2(3 \%)$

$6(9 \%)$

$15(5 \%)$

$40(14 \%)$

$9(13 \%)$

$14(20 \%)$

$4(6 \%)$

$18(26 \%)$

$19(27 \%)$

$13(23 \%)$

$17(30 \%)$

380

$28(39 \%)$

$34(12 \%)$

$64(22 \%)$

$23(8 \%)$

$77(26 \%)$

$83(28 \%)$

$45(20 \%)$

$63(28 \%)$

$90(29 \%)$

424

$6(7 \%)$
$10(12 \%)$
$15(18 \%)$
$9(11 \%)^{c}$

$22(6 \%)$

$12(4 \%)$

$44(13 \%)$

$8(2 \%)$

\begin{tabular}{l} 
\\
\\
0.7 \\
0.8 \\
0.2 \\
\\
0.4 \\
0.3 \\
0.8 \\
0.8 \\
0.5 \\
0.9 \\
0.8 \\
0.6 \\
0.7 \\
0.1 \\
\\
0.8 \\
0.002 \\
0.2 \\
0.002 \\
\hline
\end{tabular}

AED, antiepileptic drugs; EDH, epidural hemorrhage; SAH, subarachnoidal hemorrhage; SDH, subdural hemorrhage.

${ }^{a}$ Mean (s.D.).

bedian (range).

${ }^{\mathrm{C}}$ Four patients had BMI above $30 \mathrm{~kg} / \mathrm{m}^{2}$ (all diagnosed with isolated hypogonadism), five patients had BMI below $30 \mathrm{~kg} / \mathrm{m}^{2}$ (isolated GH $(n=1), \mathrm{LH} / \mathrm{FSH}(n=1)$, and prolactin $(n=2)$ deficiency and $\mathrm{GH}+$ prolactin deficiency $(n=1))$.

(71\%) had mild TBI as evaluated by the Glasgow coma scale (GCS). None of the patients were diagnosed with pituitary dysfunction before the protocol and thus none received supplemental substitution therapy. Median 2.5 (1.0-4.0) years after the index trauma, 84/426 (20\%) patients were biochemically deficient at one $(78 / 426(18 \%))$ or two (6/426 (2\%)) pituitary axes, with affection of the following: $\mathrm{GH}$ : 28/426 (6.6\%); LH/FSH: 23/426 (5.4\%); prolactin: 20/426 (4.7\%); ACTH: 17/426 (4.0\%); and TSH: 2/426 (0.5\%). None had affection of three or more axes.

Deficient patients were older and more frequently reported daily treatment with opioids and antidiabetics (Table 1). Opioid treatment was more frequent in ACTHdeficient patients ( $17 \%$ vs $4 \% ; P=0.05)$ and hypogonadal patients $(19 \%$ vs $4 \% ; P=0.002)$, whereas use of antidiabetics was more frequent in hypogonadal patients (19\% vs $4 \% ; P=0.002)$ and hypoprolactinemic patients ( $15 \%$ vs $4 \% ; P=0.06$ ). Deficient and sufficient patients did not differ as to trauma-related factors (Table 1).

\section{QoL and injury-related factors}

Patients with moderate to severe TBI (as indicated by GCS $<13$ ) had lower EQ5D VAS $(P=0.01)$, increased 'physical and mental fatigue' $(P<0.02)$, decreased 'physical' $(P=0.002)$ and 'social' $(P=0.04)$ function, 'physical' $(P=0.01)$ and 'emotional' ( $P=0.04)$ role, relative to patients with mild TBI. Patients with traumatic subarachnoid hemorrhage (SAH) had lower EQ5D VAS, 'general health', 'physical function and activity', and increased 'physical fatigue' (all $P<0.05$ ) 

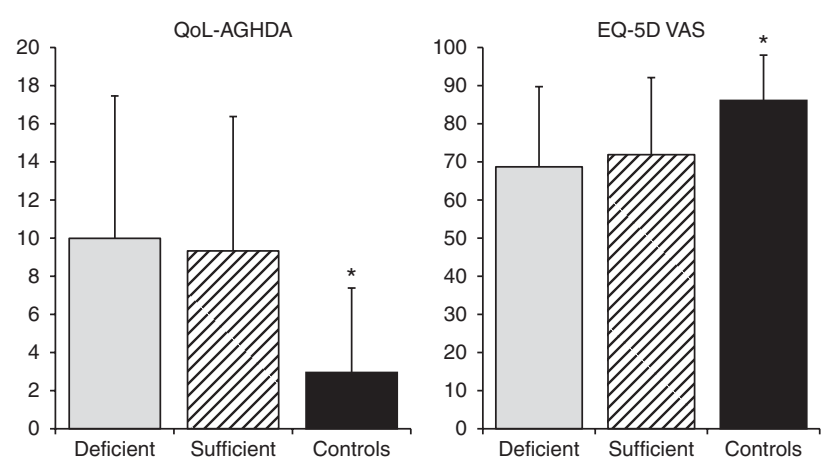

\section{Figure 1}

Mean (s.D.) EQ-5D VAS and QoL-AGHDA scores in healthy controls (black bars) and in TBI patients with pituitary deficiency (light grey bars) and sufficiency (diagonal striped bars) respectively. EQ-5D VAS measures overall health status on a visual analogue scale, scored $0-100$, with 100 denoting the best imaginable health. QoL-AGHDA measures the impact of GH deficiency in one overall score, range from 0 to 25, with higher scores representing worse HRQL. ${ }^{*} P<0.001$ compared with TBI patients (unadjusted).

relative to those without $\mathrm{SAH}$. Likewise, patients with subdural hemorrhage had increased 'general fatigue' $(P=0.01)$, 'reduced motivation', 'vitality', and 'mental health' (all $P<0.05$ ). No differences in patient reported outcome were observed in patients with and without epidural hemorrhage, vault or base of skull fractures, cerebral contusions, cerebral edema, or axonal injury.

Patients with Glasgow outcome scale $<5$ (i.e. subnormal recovery) scored worse in all scales $(P<0.0001)$.

\section{QoL and medical treatment}

In patients, treatment with antidepressants was related to worse outcome scores in all scales $(P<0.001)$. Opioid treatment was related to worse scores $(P<0.001)$ in all scales but 'reduced motivation'. Patients medically treated for diabetes had lower 'physical function' $(P=0.002)$ and increased 'physical fatigue' $(P=0.01)$, whereas those treated with antiepileptic drugs had lower EQ5D VAS $(P=0.01)$, increased 'physical' and 'mental fatigue' $(P<0.01)$, and decreased 'physical function' $(P=0.002)$ and 'physical role' $(P<0.001)$.

\section{HRQL in patients and controls}

Age- and gender-adjusted EQ5D, SF36, QoLAGHDA, and MFI-20 scores were significantly worse in both sufficient and insufficient patients, relative to healthy controls (all $P<0.0001$ ) (Figs 1 and 2).

\section{HRQL and pituitary function}

Patients with pituitary deficiencies $(n=84)$ had increased 'physical fatigue' $(P=0.03)$, worse 'physical component score' $(P=0.03)$, and 'physical function' $(P=0.01)$ relative to patients with intact pituitary function (Figs 1 and 2).
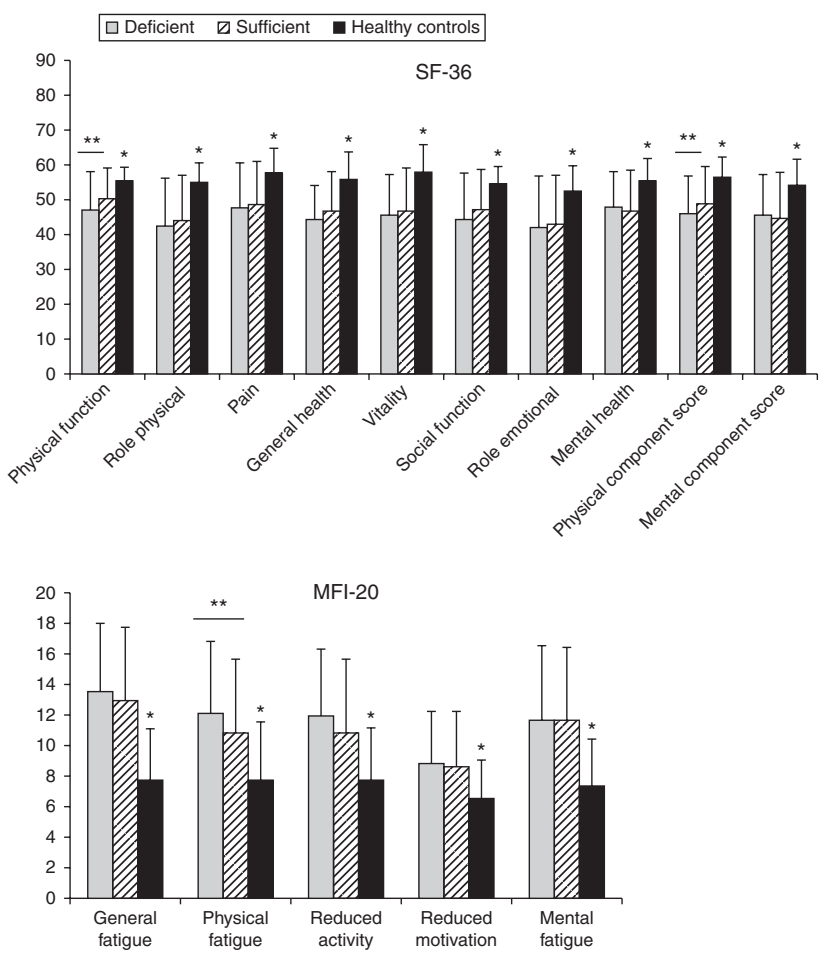

Figure 2

Mean (s.D.) SF-36 and MFI-20 scores in healthy controls (black bars), and TBI patients with pituitary deficiency (light grey bars) and sufficiency (diagonal striped bars) respectively. SF-36 consists of 36 items summated into eight scales measuring different dimensions of health. The eight scales are further condensed into two summary component scores of mental and physical health. Within the SF-36 scores, low values denote a lower self-rated general health. Data quality of the SF-36 responses was determined using QualityMetric Health Outcomes Scoring Software 4.5 (QualityMetric, Lincoln, RI, USA). MFI-20 consists of five scales based on different modes of expressing fatigue (general fatigue, mental fatigue, reduced activity, reduced motivation, and mental fatigue). Scores range from 4 to 20 , with high scores representing increased fatigue. ${ }^{*} P<0.001$ compared with both sufficient and insufficient TBI patients; $* * P<0.05$ comparing deficient and sufficient TBI patients (unadjusted). 
Gender, age, and waist circumference explained 14-21\% of the total variation in physical fatigue, function, and component score in healthy controls. Adjustment for these covariates removed the relationship between pituitary deficiency and questionnaire scores for all but 'physical function' ( $\beta=-2.5 ; P=0.03)$.

Patients with untreated hypogonadotropic hypogonadism (19 men and four women) had worse 'physical component score' $(P=0.02)$, 'physical functioning' $(P=0.004)$, and 'social functioning' $(P=0.05)$ and increased 'physical fatigue' $(P=0.05)$ compared with eugonadal patients. 'Physical function' $(\beta=-5.2$; $P=0.005)$ and 'social function' $(\beta=-5.7 ; P=0.02)$ remained significantly related to gonadal insufficiency after adjustment for gender, age, and waist circumference. Hypogonadism did not remain significantly related to any of the scale scores by further adjustment for medical treatment with opioids, antidepressants, and antidiabetic agents (Table 2).

Patients with GH deficiency $(n=28)$ had decreased overall health perception on the EQ5D VAS $(P=0.03)$, which was further strengthened after adjustment for the above covariates $\left(P=0.01 ; R^{2}=0.14\right)$ (Table 2$)$. However, $\mathrm{GH}$ deficiency was not related to any of the other scale scores, including the QoLAGHDA.

Neither ACTH, TSH nor prolactin deficiency was independently related to any of the questionnaire scores.

In univariate analysis, lower physical component score was related to lower total $(\beta=0.35 ; P<0.001)$ and free $(\beta=2.99 ; P=0.03)$ testosterone in men. Increased fatigue (general and physical), worse physical SF36 scores (physical fatigue, bodily pain, and role physical), and EQ5D VAS were significantly related to lower total testosterone but not to free testosterone. Total but not

Table 2 Multiple regression analyses on the relationship between EQ5D VAS, SF36, and MFI-20 physical scores and (A) pituitary deficiencies and (B) hormonal concentrations. Data are presented as $\beta$-coefficients. Lower coefficients denote greater impairment to HRQL (EQ5D and SF36), whereas higher coefficients denote greater fatigue (MFI-20). Multiple regression analyses with EQ5D VAS, SF36, and MFI-20 physical scores entered as the dependent variable and gender $(\operatorname{men}=1$, women $=0)$, age, waist circumference, Glasgow coma scale, medications (yes $=1$, no $=0$ ) together with either $(a)$ hypogonadotropic hypogonadism or $\mathrm{GH}$ deficiency (yes $=1$, no $=0)$, or $(b)$ testosterone or IGF1, entered as independent variables in each model.

\begin{tabular}{|c|c|c|c|c|}
\hline & \multicolumn{4}{|c|}{ Questionnaire } \\
\hline & \multirow{2}{*}{$\frac{\text { EQ-5D }}{\text { VAS }}$} & \multicolumn{2}{|c|}{ Short Form-36 } & \multirow{2}{*}{$\begin{array}{c}\text { MFI-20 } \\
\text { Physical fatigue }\end{array}$} \\
\hline & & PCS & Physical function & \\
\hline (A) Multivariate, adjusted $R^{2}$ & 0.18 & 0.16 & 0.22 & 0.18 \\
\hline Gender (women) & $-5.83^{\dagger}$ & $-3.40^{\dagger}$ & $-3.72^{\dagger}$ & $1.71^{\dagger}$ \\
\hline Age (10 years) & 1.33 & -0.28 & -0.40 & 0.03 \\
\hline Waist $(10 \mathrm{~cm})$ & $-2.36^{\dagger}$ & $-1.12^{\dagger}$ & $-0.95^{\dagger}$ & $0.67^{\dagger}$ \\
\hline GCS & $5.73^{\dagger}$ & $-2.59 *$ & $-3.54^{\dagger}$ & $1.59^{\dagger}$ \\
\hline Antidepressants & $-10.34^{\dagger}$ & $-4.87^{\dagger}$ & $-3.74^{\dagger}$ & $1.96^{\dagger}$ \\
\hline Opioids & $-18.40^{\dagger}$ & $-10.60^{\dagger}$ & $-9.15^{\dagger}$ & $4.02^{\dagger}$ \\
\hline Antidiabetics & -5.84 & -1.54 & $-4.21 *$ & $2.30 *$ \\
\hline AEDs & $-11.57^{\dagger}$ & -1.96 & -1.37 & $2.07 *$ \\
\hline Hypogonadotropic hypogonadism ${ }^{a}$ & $2.64^{P=0.58}$ & $-1.05^{P=0.67}$ & $-3.32^{P=0.10}$ & $-0.23^{P=0.84}$ \\
\hline GH deficiency & $-10.84^{P=0.01}$ & $-3.44^{P=0.09}$ & $-2.19^{P=0.22}$ & $1.54^{P=0.10}$ \\
\hline (B) Multivariate (adjusted $R^{2}$ ) & 0.14 & 0.16 & 0.20 & 0.18 \\
\hline Gender & - & - & - & - \\
\hline Age (10 years) & 0.90 & -0.50 & -0.64 & 0.05 \\
\hline Waist $(10 \mathrm{~cm})$ & -1.61 & -0.57 & -0.66 & 0.40 \\
\hline GCS & $-5.60 *$ & -1.98 & $-3.03^{\dagger}$ & $1.61 *$ \\
\hline Antidepressants & $-13.00^{\dagger}$ & $-5.13^{\dagger}$ & $-6.03^{\dagger}$ & $2.80^{\dagger}$ \\
\hline Opioids & -4.32 & $-6.63 *$ & -4.16 & -0.03 \\
\hline Antidiabetics & -7.90 & -4.79 & $-6.11^{\dagger}$ & $3.64^{\dagger}$ \\
\hline AEDs & -7.94 & -1.75 & -2.17 & 0.51 \\
\hline Testosterone & $0.25^{P=0.21}$ & $0.21^{P=0.04}$ & $0.08^{P=0.35}$ & $-0.09^{P=0.06}$ \\
\hline IGF1 & $-0.01^{P=0.85}$ & $0.003^{P=0.64}$ & $0.004^{P=0.65}$ & $-0.002^{P=0.78}$ \\
\hline
\end{tabular}

AED, antiepileptic drugs; GCS, Glasgow coma scale; VAS, visual analogue scale; PCS, physical component score. Data are presented as $\beta$-coefficients, adjusted $R^{2} ;{ }^{*} P<0.05$ and ${ }^{\dagger} P<0.001$.

${ }^{a}$ Men and women, all untreated. 
free testosterone remained related to worse 'physical component score', 'bodily pain', and 'general health perception' ( $\left.\beta=0.12-0.24 ; P<0.05 ; R^{2} 0.09-0.13\right)$ after adjustment for age, waist circumference, and medical treatment with opioids, antidepressants, and antidiabetic agents (Table 2).

Age- and gender-adjusted IGF1 was related to physical functioning ( $\beta=0.03$ and $P=0.04$ ), which did not remain after further adjustment for waist circumference (Table 2).

None of the other hormone concentrations were independently related to any of the questionnaire scores.

\section{Pituitary symptom questionnaire}

The symptoms addressed were commonly acknowledged by the patients, with a particularly high prevalence of fatigue $(>60 \%)$, changed sleep pattern $(>50 \%)$, and decreased libido $(>25 \%)$. Decreased libido $(P=0.03)$, erectile function $(P=0.03)$, and hair growth $(P=0.01)$ and increased fatigue $(P=0.01)$ and sleep pattern changes $(P=0.03)$ were more commonly acknowledged by deficient compared with sufficient men. Only decreased hair growth was independently related to male hypogonadotropic hypogonadism $(P=0.01$; adjusted for age, waist circumference, and medical treatment with opioids, antidepressants, and antidiabetic agents). The frequency of acknowledged symptoms was similar in deficient and sufficient women.

Use of antidepressants was the strongest predictor for decreased libido $(P=0.001)$, erectile function $(P<0.001)$, and fatigue $(P=0.01)$, whereas GCS $<13$ was the strongest predictor for change in sleep pattern.

\section{Discussion}

In a population-based TBI cohort, we explored different aspects of patient reported outcome in relation to pituitary deficiency.

Compared with matched controls, patients with TBI had significant and substantial detriments in their QoL and symptom scores. Although controversies may exist concerning the disabilities associated with mild TBI (18), it is generally agreed that survivors of moderate-to-severe TBI are at increased risk of various degrees of permanent disability. Our cohort included patients with mild, moderate, and severe TBI and still showed QoL impairment, sleep disturbances, fatigue, depression, and sexual dysfunction at comparable rates to previous reports (19).

Patients with pituitary deficiencies had increased impairment of various mainly physical scales relative to patients with intact pituitary function. These differences were only partially confirmed upon adjustment for differences in gender, age, and waist circumference, in keeping with previous observations that gender, age (15), and body composition (20) have to be considered to avoid considerable bias analyzing QoL data.

As pituitary deficiencies may affect QoL differently, axis-specific relations were sought for. Patients with hypogonadotropic hypogonadism (men and women) had worse physical and social functioning and increased physical fatigue compared with eugonadal patients. Our data also suggested increasing impairments with declining total testosterone concentrations in men. However, the observed relations were only modest, and overall not reproduced for free testosterone. Consistently, androgen deficiency was neither identified as the sole nor the most important cause of impaired QoL and fatigue in TBI patients, after adjustment for significant covariates including medication known to influence endocrine pathways.

Impaired QoL and fatigue are common in androgen deficiency (21). Androgen deficiency is, however, related to several comorbidities including obesity, diabetes, depression, and opioid treatment, all of which are independently related to decreased QoL $(22,23,24)$. In healthy elderly men, bioavailable testosterone was only modestly associated with QoL, whereas more closely associated with central fat mass (20). In our study, additional to age and waist circumference, medication with antidepressants, opioids, anticonvulsants, and antidiabetics were more closely associated with QoL and fatigue than total and free testosterone levels. Decreased testosterone concentrations in obesity and diabetes mellitus are linked to decreased SHBG (21), whereas opioids may cause inhibition at the hypothalamic, pituitary, and end-organ level (25). Anticonvulsants used in TBI patients for treatment of pain and epilepsy may among other influences increase testosterone metabolism via induction of hepatic enzymes in men (26). Lower testosterone levels in depressive illness are probably linked to activation of the general stress response (27), which in turn may be responsible for down-regulated testosterone biosynthesis in the Leydig cell (28). Consequently, since testosterone concentrations are closely associated with age, body composition, and above medications, it may not have an independent effect on QoL. However, as many of the covariates display a mutual relationship, it may be difficult to discern which dysfunction is causal if any. While only total but not free testosterone was independently related to QoL in this study, it may theoretically remain an important and treatable syndrome in selected 
patients. Hypogonadism has complex physiology and relating testosterone concentrations to QoL may be too simplified, as androgen receptor polymorphisms, and posttranscriptional factors cannot be taken into account in such analyses.

Patients with GH deficiency had worse EQ5D VAS scores, whereas the QoLAGHDA score specific to $\mathrm{GH}$ deficiency did not differ from patients with intact $\mathrm{GH}$ secretion. IGF1 levels did not relate to QoLAGHDA or any other scale scores. Decreased QoL including lowering of the QoLAGHDA score was previously reported in posttraumatic GH deficiency $(5,7)$. The reason that we were unable to identify such relations in the present cohort is unclear. A possible explanation is the high risk of irreproducible GH test outcomes $(4,29)$, which could have led to misdiagnosis (false positive), thus blurring the statistical analyses, not only in the present but also in all previous studies relying on single testing.

QoL is increasingly used for patient assessment and treatment evaluation. It is a complex entity integrating the patient's physical, mental, and social well-being, and due to its nature susceptible to many often coexisting conditions. In this study, medical treatment by antidepressant, opioids, and antiepileptic drugs reflected persistent physical and mental sequelae to the index trauma, which may be more important for the QoL than any hormonal deficiency per se. Symptom clusters including fatigue, depression, pain, and sleep disorders strongly predict lower QoL in various disease states other than pituitary deficiency $(30,31)$, which might explain the modest or even absent associations between hormonal deficiencies and QoL after adjustment for above covariates with a possible overpowering dominant influence.

Some concerns related to the study should be addressed. First, this is an observational study from which causality cannot be inferred. Due to the dominant effect of trauma sequelae and coexisting morbidities often mutually related to hormonal deficiencies, causality may remain difficult to prove. Until now, data on treatment effect in posttraumatic hypopituitarism remain limited and comes from small non-randomized intervention studies $(8,32)$ and reports from the KIMS database (33).

Second, only indirect information on depression and diabetes was obtained from the use of medications at the time of testing. The prevalence of depression in the Danish background population is $3-4 \%$, but considerably increased after TBI (34); $14 \%$ of our patients were treated with antidepressants, which is in keeping with previous reports (35), indicating that severe underestimation is unlikely. Further, only patients receiving antidiabetics fulfilled the diagnostic criteria for diabetes mellitus.

Yet another concern could be a potential invalidity of the posttraumatic QoL scores. The cerebral lesions in TBI patients often involve the frontal lobe region, and therefore the patients may lack awareness and insight into their own dysfunctions. QoL items reporting of these dysfunctions may consequently be underestimated (36). As posttraumatic QoL scores from significant others (closest relatives) were only random, we were not able to correct for this potential bias.

The primary strength of this study is its size and standardized protocol ensuring fasting morning sampling in well characterized patients enabling appropriate adjustment for significant comorbidities. Further, all samples from healthy controls and patients were frozen and analyzed by the same assays within few batches. Total testosterone was determined by the gold standard reversephase chromatography with tandem mass spectrometry, from which free testosterone was calculated. Local assayspecific cutoff values were calculated from healthy controls further minimizing the risk of methodological bias. Cutoff values generally resembled those from the literature, although peak cortisol in response to ITT was significantly lower than that usually applied, in keeping with previous reports $(37,38)$. Accordingly, $15 \%$ of our controls failed the general cutoff of $500 \mathrm{nmol} / \mathrm{l}$. Falsepositive cases were also acknowledged by Vestergaard et al. (37), who described peak cortisol during ITT ranging from 448 to $775 \mathrm{nmol} / \mathrm{l}$ in healthy controls. Also, Simsek et al. (38) recently reported significantly lower peak cortisol during ITT compared with ACTH and glucagon tests in hypopituitary patients and defined a local peak cortisol response for the ITT of $<300 \mathrm{nmol} / 1$ for determining adrenal failure. As to the appropriateness of the applied cutoff value in the present cohort, 12 of 208 (6\%) patients undergoing ITT were diagnosed as sufficient, although failing a cutoff value of $500 \mathrm{nmol} / \mathrm{l}$; ten had a $30 \mathrm{~min}$ plasma cortisol ranging from 540 to $770 \mathrm{nmol} / \mathrm{l}$ in response to synacthen stimulation, whereas two patients might have been misclassified as they had insufficient 30 min plasma cortisol concentrations of 481 and $486 \mathrm{nmol} / 1$ respectively. On the other hand, five patients had a peak cortisol below $398 \mathrm{nM}$, but a normal synacthen test with a 30 min cortisol ranging between 589 and $718 \mathrm{nmol} / \mathrm{l}$, which underline the complexity of the diagnosis where in some cases the clinical context and repeated testing has to be considered for the final decision as to whether or not a patient is considered insufficient. Cutoff values always reflect the balance 
between test sensitivity and specificity, and have to be addressed in the clinical context. The use of $398 \mathrm{nmol} / \mathrm{l}$ in the present study was justified by assessment in a lowrisk population, although a risk of diagnostic uncertainty is acknowledged.

In conclusion, compared with matched controls, patients with TBI have significant and substantial detriments in their QoL. The relationship between pituitary hormone deficiencies upon single unconfirmed testing and QoL was modest compared with that of various TBI sequelae and related non-hormonal comorbidities. For patients with TBI, our results suggest that the presence of concurrent comorbidities plays a significant role in QoL and that identifying these comorbidities should be of greater priority than focus on pituitary function. Causality is still to be shown, and whether hormonal substitution therapy could be of additional relevance in cases of mild deficiencies needs to be proven. However, in cases of overt posttraumatic multiple pituitary hormone deficiencies, treatment effect is expected of similar efficacy as in any other cause of pituitary deficiency and thus needs to be considered.

\section{Declaration of interest}

The authors declare that there is no conflict of interest that could be perceived as prejudicing the impartiality of the research reported.

\section{Funding}

The study was funded by unrestricted grants from: The Danish Agency for Science, Technology and Innovation, The Research Council of the Capital Region of Denmark, The Lundbeck Foundation, Novo Nordic, The A.P. Moller Foundation for the Advancement of Medical Science, Arvid Nilsson's Foundation, Christenson-Ceson's Foundation, Axel-Muusfeldt's Foundation and Else and Mogens Wedell-Wedellsborg's Foundation. Immunodiagnostic Systems provided iSYS GH kits free of charge, but had no influence on results or their interpretation. The study was registered at ClinicalTrials.gov NCT01028742.

\section{Author contribution statement}

M Klose made primary contributions to data collection and analysis, interpretation of results, and writing of the manuscript. M Klose, $\mathrm{U}$ Feldt-Rasmussen, J S Christiansen, M Andersen, $\mathrm{P}$ Laurberg, and $K$ Stochholm contributed to the study conception and design. J Janukonyté and L L Christensen contributed to data collection, A S Cohen and A Wagner contributed to data collection/analyses. All authors contributed to interpretation of results, all revised the manuscript critically for important intellectual content and all approved the final manuscript. M Klose is the guarantor.

\section{Acknowledgements}

The authors thank laboratory technicians Casper Kok, Anette Vinding, Kirsten Rasmussen, Susanne Andersen, and Anne-Mette Christensen for their excellent technical assistance. They are also grateful to Pfizer (M Koltowska-Haggstrøm) for the permission to use QoLAGHDA in the present study.

\section{References}

1 Molitch ME, Clemmons DR, Malozowski S, Merriam GR \& Vance ML. Evaluation and treatment of adult growth hormone deficiency: an Endocrine Society clinical practice guideline. Journal of Clinical Endocrinology and Metabolism 201196 1587-1609. (doi:10.1210/jc. 2011-0179)

2 Heather NL, Jefferies C, Hofman PL, Derraik JG, Brennan C, Kelly P, Hamill JK, Jones RG, Rowe DL \& Cutfield WS. Permanent hypopituitarism is rare after structural traumatic brain injury in early childhood. Journal of Clinical Endocrinology and Metabolism 201297 599-604. (doi:10.1210/jc.2011-2284)

3 Kokshoorn NE, Smit JW, Nieuwlaat WA, Tiemensma J, Bisschop PH, Groote VR, Roelfsema F, Franken AA, Wassenaar MJ, Biermasz NR et al. Low prevalence of hypopituitarism after traumatic brain injury: a multicenter study. European Journal of Endocrinology 2011165 225-231. (doi:10.1530/EJE-11-0365)

4 Klose M, Stochholm K, Janukonyte J, Lehman CL, Frystyk J, Andersen M, Laurberg P, Christiansen JS \& Feldt-Rasmussen U. Prevalence of posttraumatic growth hormone deficiency is highly dependent on the diagnostic set-up: results from The Danish National Study on Posttraumatic Hypopituitarism. Journal of Clinical Endocrinology and Metabolism 201499 101-110. (doi:10.1210/jc.2013-2397)

5 Klose M, Watt T, Brennum J \& Feldt-Rasmussen U. Posttraumatic hypopituitarism is associated with an unfavorable body composition and lipid profile, and decreased quality of life 12 months after injury. Journal of Clinical Endocrinology and Metabolism 200792 3861-3868. (doi:10.1210/jc.2007-0901)

6 Bavisetty S, Bavisetty S, McArthur DL, Dusick JR, Wang C, Cohan P, Boscardin WJ, Swerdloff R, Levin H, Chang DJ et al. Chronic hypopituitarism after traumatic brain injury: risk assessment and relationship to outcome. Neurosurgery 200862 1080-1093. (doi:10. 1227/01.neu.0000325870.60129.6a)

7 Kelly DF, McArthur DL, Levin H, Swimmer S, Dusick JR, Cohan P, Wang C \& Swerdloff R. Neurobehavioral and quality of life changes associated with growth hormone insufficiency after complicated mild, moderate, or severe traumatic brain injury. Journal of Neurotrauma 2006 23 928-942. (doi:10.1089/neu.2006.23.928)

8 Nourollahi S, Wille J, Weiss V, Wedekind C \& Lippert-Gruner M. Quality-of-life in patients with post-traumatic hypopituitarism. Brain Injury 201428 1425-1429. (doi:10.3109/02699052.2014.917201)

9 Bondanelli M, Ambrosio MR, Cavazzini L, Bertocchi A, Zatelli MC, Carli A, Valle D, Basaglia N \& Uberti EC. Anterior pituitary function may predict functional and cognitive outcome in patients with traumatic brain injury undergoing rehabilitation. Journal of Neurotrauma 200724 1687-1697. (doi:10.1089/neu.2007.0343)

10 Pavlovic D, Pekic S, Stojanovic M, Zivkovic V, Djurovic B, Jovanovic V, Miljic N, Medic-Stojanoska M, Doknic M, Miljic D et al. Chronic cognitive sequelae after traumatic brain injury are not related to growth hormone deficiency in adults. European Journal of Neurology 201017 696-702. (doi:10.1111/j.1468-1331.2009.02910.x)

11 Nielsen TL, Hagen C, Wraae K, Brixen K, Petersen PH, Haug E, Larsen R $\&$ Andersen $\mathrm{M}$. Visceral and subcutaneous adipose tissue assessed by magnetic resonance imaging in relation to circulating androgens, sex hormone-binding globulin, and luteinizing hormone in young men. Journal of Clinical Endocrinology and Metabolism 200792 2696-2705. (doi:10.1210/jc.2006-1847)

12 Klose M, Lange M, Rasmussen AK, Skakkebaek NE, Hilsted L, Haug E, Andersen M \& Feldt-Rasmussen U. Factors influencing the adrenocorticotropin test: role of contemporary cortisol assays, body 
composition, and oral contraceptive agents. Journal of Clinical Endocrinology and Metabolism 200792 1326-1333. (doi:10.1210/ jc.2006-1791)

13 Bartsch W. Interrelationships between sex hormone-binding globulin and testosterone, $5 \alpha$-dihydrotestosterone and oestradiol-17 $\beta$ in blood of normal men. Maturitas 19802 109-118. (doi:10.1016/ 0378-5122(80)90044-4)

14 EuroQol Group. EuroQol - a new facility for the measurement of health-related quality of life. Health Policy 199016 199-208. (doi:10.1016/0168-8510(90)90421-9)

15 Ware JE \& Kosinski M. SF-36 Physical and Mental Health Summary Scales: A Manual For Users of Version 1, 2nd end, 2001

16 McKenna SP, Doward LC, Alonso J, Kohlmann T, Niero M, Prieto L \& Wiren L. The QoL-AGHDA: an instrument for the assessment of quality of life in adults with growth hormone deficiency. Quality of Life Research 19998 373-383. (doi:10.1023/A:1008987922774)

17 Smets EM, Garssen B, Bonke B \& De Haes JC. The Multidimensional Fatigue Inventory (MFI) psychometric qualities of an instrument to assess fatigue. Journal of Psychosomatic Research 199539 315-325. (doi:10.1016/0022-3999(94)00125-O)

18 Ruff RM. Mild traumatic brain injury and neural recovery: rethinking the debate. NeuroRehabilitation 201128 167-180. (doi:10.3233/NRE2011-0646)

19 Forslund MV, Roe C, Sigurdardottir S \& Andelic N. Predicting healthrelated quality of life 2 years after moderate-to-severe traumatic brain injury. Acta Neurologica Scandinavica 2013128 220-227. (doi:10.1111/ ane.12130)

20 Glintborg D, Nielsen TL, Wraae K, Hougaard D, Gudex C, Brixen K \& Andersen M. The relationship between health-related quality of life, obesity and testosterone levels in older men. Age and Ageing 201443 280-284. (doi:10.1093/ageing/aft203)

21 Bhasin S, Cunningham GR, Hayes FJ, Matsumoto AM, Snyder PJ Swerdloff RS \& Montori VM. Testosterone therapy in men with androgen deficiency syndromes: an Endocrine Society clinical practice guideline. Journal of Clinical Endocrinology and Metabolism 201095 2536-2559. (doi:10.1210/jc.2009-2354)

22 Rubin RR \& Peyrot M. Quality of life and diabetes. Diabetes/Metabolism Research and Reviews 199915 205-218. (doi:10.1002/(SICI)15207560(199905/06)15:3<205::AID-DMRR29> 3.0.CO;2-O)

23 Sjogren P, Gronbaek M, Peuckmann V \& Ekholm O. A population-based cohort study on chronic pain: the role of opioids. Clinical Journal of Pain 201026 763-769. (doi:10.1097/AJP.0b013e3181f15daf)

24 Apovian CM. The clinical and economic consequences of obesity. American Journal of Managed Care 201319 s219-s228.

25 Morley JE. The endocrinology of the opiates and opioid peptides. Metabolism 198130 195-209. (doi:10.1016/0026-0495(81)90172-4)

26 Bauer J, Blumenthal S, Reuber M \& Stoffel-Wagner B. Epilepsy syndrome, focus location, and treatment choice affect testicular function in men with epilepsy. Neurology 200462 243-246. (doi:10.1212/01.WNL.0000091866.48962.79)
27 Zitzmann M \& Nieschlag E. Testosterone levels in healthy men and the relation to behavioural and physical characteristics: facts and constructs. European Journal of Endocrinology 2001144 183-197. (doi:10.1530/eje.0.1440183)

28 Hardy MP \& Ganjam VK. Stress, 11ß-HSD, and Leydig cell function. Journal of Andrology $1997 \mathbf{1 8}$ 475-479.

29 Lissett CA, Thompson EG, Rahim A, Brennan BM \& Shalet SM. How many tests are required to diagnose growth hormone (GH) deficiency in adults? Clinical Endocrinology 199951 551-557. (doi:10.1046/j.13652265.1999.00836.x)

30 Reyes-Gibby CC, Aday LA, Anderson KO, Mendoza TR \& Cleeland CS. Pain, depression, and fatigue in community-dwelling adults with and without a history of cancer. Journal of Pain and Symptom Management 200632 118-128. (doi:10.1016/j.jpainsymman.2006.01.008)

31 Naess H, Lunde L \& Brogger J. The effects of fatigue, pain, and depression on quality of life in ischemic stroke patients: the Bergen Stroke Study. Vascular Health and Risk Management 20128 407-413. (doi:10.2147/VHRM.S32780)

32 Moreau OK, Cortet-Rudelli C, Yollin E, Merlen E, Daveluy W \& Rousseaux M. Growth hormone replacement therapy in patients with traumatic brain injury. Journal of Neurotrauma 201330 998-1006. (doi:10.1089/neu.2012.2705)

33 Kreitschmann-Andermahr I, Poll EM, Reineke A, Gilsbach JM, Brabant G, Buchfelder M, Fassbender W, Faust M, Kann PH \& Wallaschofski $\mathrm{H}$. Growth hormone deficient patients after traumatic brain injury - baseline characteristics and benefits after growth hormone replacement - an analysis of the German KIMS database. Growth Hormone \& IGF Research 200818 472-478. (doi:10.1016/j.ghir. 2008.08.007)

34 Orlovska S, Pedersen MS, Benros ME, Mortensen PB, Agerbo E \& Nordentoft $\mathrm{M}$. Head injury as risk factor for psychiatric disorders: a nationwide register-based follow-up study of 113,906 persons with head injury. American Journal of Psychiatry 2014171 463-469. (doi:10.1176/appi.ajp.2013.13020190)

35 Sullivan-Singh SJ, Sawyer K, Ehde DM, Bell KR, Temkin N, Dikmen S, Williams RM \& Hoffman JM. Comorbidity of pain and depression among persons with traumatic brain injury. Archives of Physical Medicine and Rehabilitation 201495 1100-1105. (doi:10.1016/j.apmr.2014.02.001)

36 Sbordone RJ, Seyranian GD \& Ruff RM. Are the subjective complaints of traumatically brain injured patients reliable? Brain Injury 199812 505-515. (doi:10.1080/026990598122467)

37 Vestergaard P, Hoeck HC, Jakobsen PE \& Laurberg P. Reproducibility of growth hormone and cortisol responses to the insulin tolerance test and the short ACTH test in normal adults. Hormone and Metabolic Research 199729 106-110. (doi:10.1055/s-2007-979000)

38 Simsek Y, Karaca Z, Tanriverdi F, Unluhizarci K, Selcuklu A \& Kelestimur F. A comparison of low-dose ACTH, glucagon stimulation and insulin tolerance test in patients with pituitary disorders. European Journal of Endocrinology 201582 45-52.

Received 5 December 2014

Revised version received 10 March 2015

Accepted 12 March 2015 